\title{
Teacher candidates' comprehension of first and high order quantifiers
}

\author{
PInar ANAPA SABAN \\ Eskişehir Osmangazi University, Education Faculty, Primary Education Department, Turkey. \\ Received 10 July, 2014; Accepted 27 August, 2014
}

\begin{abstract}
The purpose of this study was to investigate the quantifier structuring processes of teacher candidates doing the Abstract Mathematics course of the Elementary School Mathematics Teaching program. Educational activities assisting the students' structuring of quantifiers were provided through transitions between verbal (written) expressions and symbolic expression in open statements that contained first, second, and third order quantifiers. The data of this study, based on an APOS (Action, Process, Object, and Schema) theoretical framework, were obtained by using a problem form. This form consists of four questions on quantization prepared by the researcher to investigate the cognitive development of the students; clinical interviews were performed at the end of the study period with five students. At the end of the study, it was determined that the students were unable to fully understand expressions regarding first and high order quantifier structures, and that they developed opinions according to their own perceptions. Furthermore, important results were obtained during the study that demonstrated the level of comprehension of students regarding first, second, and third order quantifier structures.
\end{abstract}

Key words: The quantifier concept, universal quantifier, existential quantifier, genetic decompositions, APOS.

\section{INTRODUCTION}

"I think therefore I am." While searching for the truth, the famous mathematician and philosopher Descartes determined that the expression, "I think therefore I am" is the embodiment of an unshakable, solid, and reliable truth, and hence considered it without hesitation as the first principle of the philosophical system he was building [Descartes, 2013]. According to this principle, the ability to think is both proof of mankind's existence and his greatest strength. In science, we witness mankind seeking and finding truths in the universe due to his curiosity and ability to think. The key to this success of mankind is also due to another product born from mankind's thoughts and creativity: mathematics. This product is so exceptional that it has always been clearly understood by societies using different languages, and even peoples living in different time periods. This ability has made mathematics "the queen of science." The strength of mathematics ultimately stems from its own language. The main elements of the language of mathematics are quantifiers, which are designated as either universal or existential.

Quantifiers have an undeniable importance in giving meaning to mathematical information. Furthermore, the

\section{E-mail: panapa@ogu.edu.tr.}

Authors agree that this a ricle rema in pemanently open access under the tems of the $\underline{\text { Creative Commons }}$ Attribution License 4.0 Intemational Lic ense 
ability to work with quantifiers is important not only to be able to obtain mathematical information, but also to effectively using the wide range of conceptual structures in mathematics (Dubinsky et al., 1998). On the other hand, it is known that students receiving secondary education and university education experience considerable difficulties in understanding quantifiers.

Mathematical expressions that include existential and universal quantifiers are frequently used in undergraduate university courses. Undergraduate students first encounter mathematical expressions consisting of multiple quantifiers while covering the subject of limit and continuity during the "Analysis I" course. Students who do more advanced mathematics courses have to work even more extensively with such expressions (for example, while learning fundamental concepts in advanced analysis, abstract algebra and topology, such as derivatives, linear independence, coset, normal subgroups, compactness, etc...). Although students frequently use expressions with multiple quantifiers, they generally have difficulties understanding them. This difficulty experienced by the students adversely affects their ability to comprehend and prove mathematical notions. For this reason, it is rather important to determine how students define quantifiers, and to also identify the underlying reasons for the definitions they provide. Dubinsky et al. (1998) described that due to the quantifier-rich nature of undergraduate level university mathematics courses, it is necessary to determine the method of teaching that will allow students to better comprehend quantifiers, as well as the approaches that will assist their ability to give meaning to advanced mathematical notions. However, the number of studies in the literature regarding quantifiers is relatively limited.

Tall and Chin (2002) described that when defining reflexivity (one of the properties of the equivalence relation), students generally disregarded its role as a universal quantifier. In other studies, Dubinsky et al. investigated students' ability to comprehend compound sentences/expressions containing multiple order quantifiers (Dubinsky, 1997; Dubinsky et al., 1988), and concluded that students had difficulties negating these expressions. Dubinsky (1997) also determined that using ISETL computer programs, when teaching quantifiers, improved the students' ability to give meaning to expressions containing quantifiers, as well as their ability to work with quantifiers.

Dubinsky and Yiparaki (2000) conducted a study in which they investigated the way students gave meaning to expressions consisting of two quantifiers, when one was universal and the other existential. In their study, they also attempted to determine how students interpreted and defined in mathematics and daily life expressions that contained universal-existential quantifiers $(\forall \exists)$ (in the mentioned sequence and existential-universal quantifiers (also in the mentioned sequence). Thus, they aimed to identify whether different methods of thinking assumed by the students assisted their learning of quantifiers in mathematics. However, they determined that from the students' perspective, examples from situations in daily life were not an effective means of giving meaning to quantifiers in mathematics. Moreover, it was determined that students did not know the difference between universal-existential quantifiers $(\forall \exists)$ and existentialuniversal quantifiers $(\exists \forall)$. The students were more willing to interpret and define English expressions that included universal-existential quantifiers than expressions that included existential-universal quantifiers $(\exists \forall)$.

Piatek-Jimenes (2010) investigated the reasoning used by students when interpreting mathematical expressions consisting of both existential and universal quantifiers, and also evaluated the factors that affected this reasoning. They also determined in their study that the matching of two similar expressions that differed only in the sequence of their quantifiers was difficult for the students, often leading to conflicts in their reasoning. As such, students had difficulties with expressions containing multiple quantifiers in which the existential quantifier came before the universal quantifier. Based on these observations, it was concluded that although mathematical expressions with multiple quantifiers are frequently encountered in advanced mathematics courses, the students had many difficulties in interpreting and proving these expressions.

The current study investigated the students' structuring of quantifiers in mathematics. The study included, at the same time, distinct theoretical, observational, and practical components. The study included a theoretical aspect with regards to the evaluation of mental frameworks that allowed the learning of the quantifier concept, and the epistemology related to these concepts. The study included an experimental aspect with regards to the assessment of whether mental frameworks assisted students in their learning. Finally, the study also included a practical aspect with regards to the evaluation of how students developed mental frameworks when learning the quantifier concept in mathematics, and the identification of difficulties experienced by the students when forming these frameworks.

Prior to more detailed explanations regarding the roles of quantifiers in giving meaning in mathematics, definitions regarding the concepts of open statements and quantifiers will first be provided.

\section{Open statements (statement-valued function) and quantifiers}

All fields of scientific research - whether they are empirical studies performed on certain cases or formal studies regarding abstract relationships - are based on a system of logic (Yıldırım, 2011). The building blocks of these systems of logic are known as "statements." In mathematics, statements are expressions that are used 
to determine validity or invalidity. Furthermore, expressions that contain at least one variable, and in which the validity or invalidity of the expression depends on the objects that are assigned to the variables, are known as "open statements." If an open statement is dependent on the variables $x, y, z, \ldots .$. , it will be shown with the symbols $p(x, y, z, \ldots \ldots)$.

When an open statement of $p(x)$ and a set of $A$ are given, the expression " $\forall x, p(x)$ " means that " $(x)$ is valid for every $x$ element of $A$ ". Here, the symbol $\forall$ can be used for the words "every $x$," "each single $x$ " and "any $x$;" it is thus called a "universal quantifier." The expression " $\exists x, p(x)$ means that " $p(x)$ is valid for at least one $x$ element of $A$." Here, the symbol " $\exists$ " can be used for the words "at least one $x$," or " some $x$;" it is thus called an "existential quantifier."

\section{The importance of quantifiers in understanding mathematics}

Aside from the conflicts and differences in the philosophical opinions of professional and amateur mathematicians regarding the nature of mathematics, there is no consensus regarding the use of quantifiers in advanced mathematics. Quantifiers play an important role in giving meaning to advanced mathematical concepts, and in ensuring that new mathematical concepts can be accepted without leading to doubts or uncertainty.

In advanced mathematical studies, there are no subjects or areas in which quantifiers are not used. Quantifiers are particularly important for defining the concepts belonging to a mathematical theory, for determining the relationship between concepts, and for making sense of a theory. For example, the definition of the unit element and the axiom of the inverse element (both of which are part of the group theory) are composed of two quantifiers. These two concepts are used in abstract algebra, and play an important role in giving meaning to concepts in many branches of mathematics such as geometry. The sequence in which quantifiers are used is important for properly understanding the definition of the unit element and the axiom of the inverse element. In the definition, the existential quantifier comes first, followed by the universal quantifier; while in the axiom, the universal quantifier comes first, followed by the existential quantifier. Failure on the students' part to notice this structure will result in an inability to understand the group theory even at its initial stages.

According to Dubinsky and Yiparaki (2000), rather than focusing on good analogies and explanations when teaching mathematics, emphasis should be placed more on ensuring that students understand what quantifiers are, and that they learn how they can use them to form complex mental images.

\section{Study purpose}

This study investigated how students used the quantifier concept in the Abstract Mathematics course of the Elementary School Mathematics Teaching program. When structuring the quantifier concept, a new statement containing a universal or existential quantifier is obtained by determining the truth values of the elements of a statement set. Following this, new statements are obtained by combining two or more quantifiers with complex mathematical statements. The formation of quantifier concepts by the students was investigated during the Abstract Mathematics course by evaluating the developments in their ability to form quantifier concepts. During the process of forming the quantifier concept, answers were sought to the following questions by taking into account that the statements were part of the prerequisite information of the algebraic and singlevariable function concept:

1. What is the relationship between the formation of quantifier concepts by the students and the verbal and symbolic representations of the statements?

2. What is the relationship between the formation of quantifier concepts by the students and their knowledge regarding single and multiple variable functions?

\section{THEORETICAL FRAMEWORK}

The APOS (Action, Process, Object, and Schema) theoretical framework was used within the context of this study. APOS is a special framework used at the undergraduate level for developing and studying programs.

The special mental structures used during the learning process of a concept are called "genetic decomposition." The APOS study framework aims to determine the genetic decomposition of the relevant concepts. The APOS study framework consisted of three components, which were theoretical analysis, teaching and the evaluation of the students' learning.

First of all, a theoretical analysis was performed by using the learning theory, the epistemology of the concept found in previous studies in the literature, and the mathematical knowledge of the researchers. The aim of this theoretical analysis was to determine the genetic decomposition or the cognitive model. Genetic decomposition refers to the mental structures a student is obliged to develop in order to learn the concept in a manner that is understandable for him/her. These mental structures are called action, process, object, and schema.

After determining the initial form of the genetic decomposition, the education process is planned and implemented. Various data collection tools are used to investigate which mental structures are formed by the students. 


\section{Theoretical analysis of quantifiers}

Prior to evaluating the genetic decomposition of quantifiers, let us consider a statement regarding the nature of mathematical knowledge, and on how it is internalized:

Internalized mathematical knowledge refers to a student's response to a problem encountered in daily life; to the actions, processes and objects regarding a problem; and to the structuring and restructuring of these actions, processes and objects by organizing them according to a certain scheme.

From this statement, it is understood that internalized mathematical knowledge refers to the coherent and compatible restructuring of actions, processes, objects, and schemes that represent mental structures regarding a particular mathematical problem.

According to the APOS theory, action is a transformation performed individually on mathematical objects according to a certain algorithm. Based on this transformation, the action is perceived by the subject as an external stimulus. For this reason, the action is also considered as a reaction of the subject to the stimulus. The action tends to control the individual. To further clarify this, let us consider the statement "Every member of my family is a teacher." If the individual considers the validity of the statement only with respect to certain individuals within his/her family, the comprehensibility of the statement is part of the action process. In this case, there is no indication that the individual considers whether the statement is valid for all members of his/her extended family, because the individual is actually focusing only on whether certain persons in his/her family are teachers.

If the individual can apply an internal process similar to the one he/she applied to the transformations (and can also reflect this on the action), then the action is considered to be internalized into the process. In this respect, the individual establishes control on the action, and can perform the action to a greater extent by using the images of the transformation and without applying the action openly. In the given example, it is possible to determine the validity or invalidity of the expression by controlling the summary of all obtained data - that is, by checking for every individual in the person's family (or, in someone else's family) whether they are actually teachers.

When the individual thinks in depth regarding the actions applied onto this process, he becomes aware of the process as a whole, notices his ability to move onto the process (either in action or in process), and can actually establish these types of transformations; the individual is then considered to have adopted the process as an object of thought. To be able to consider the actions in greater depth with regards to the process, the individuals must first encapsulate to ensure that the process becomes an entity or an object. In many mathematical processes, an object must be formed as a result of the encapsulation of the process, and work must be conducted with the process that created the object.

Looking back at the previous example, the individual can choose to negate the relevant expression, and think that it is valid for one family, but not for another. Thus, the expression will become an object with different situations that are dependent on the family in question. On the other hand, there is a certain claim for each family that is either valid or invalid.

In conclusion, a scheme is a collection that is in agreement with objects, processes, and previously formed schemes.

\section{The levels of comprehension of quantifiers in the APOS learning theory}

The genetic decomposition of quantifiers as described by Dubinsky et al. is shown below in Figure 1 [1991].

In the genetic decomposition of quantifiers, basic mental objects are simple statements that express a judgment on whether something is correct or incorrect. First, a set consisting of simple statements is defined. By using the function schemes necessary for the learning of the quantifier concept, the variables for obtaining open statements are defined. This is interpreted as the process. This means that the individual can think about the reiterations obtained in the domain by checking the validity or invalidity of the statement for each value in the domain of the function. For example, let us define the statement $p(x): x+2 \geq 3$ " on the integer set. In the expression $p, x$ will be used, which will provide the statement

set

$P=\{\ldots \ldots p(-3), p(-2), p(-1), p(0), p(1), p(2), p(3), \ldots \ldots$. for each integer.

Here, the expression $p$ is actually considered as a statement-valued function defined on a set of integers. The $P$ set is an image set of the $p$ function. The image set represents statements with a truth value of 1 for integers $\geq 1$, and a truth value of 0 for integers $<1$. Following these stages, it is determined that the $p$ statement valued function is correct for some of the integer values. This situation is expressed as "for at least one $x$ integer value, $p(x)$ is valid." Here, the expression "at least" indicates an existential quantifier. At the end of this process, the symbolic expression " $\exists x, p(x)$ " is obtained.

Secondly, there is the combination of two or more statements with standard logical connections that are either connective or divisive. Thus, a mental process is obtained through the combination of mental actions applied to the statements, and the internalization of the mental actions.

When the two processes described above are structured as necessary, the student is then ready to bring the structure to the stage of first order quantifiers.

At this order, statements include only a single quantifier 


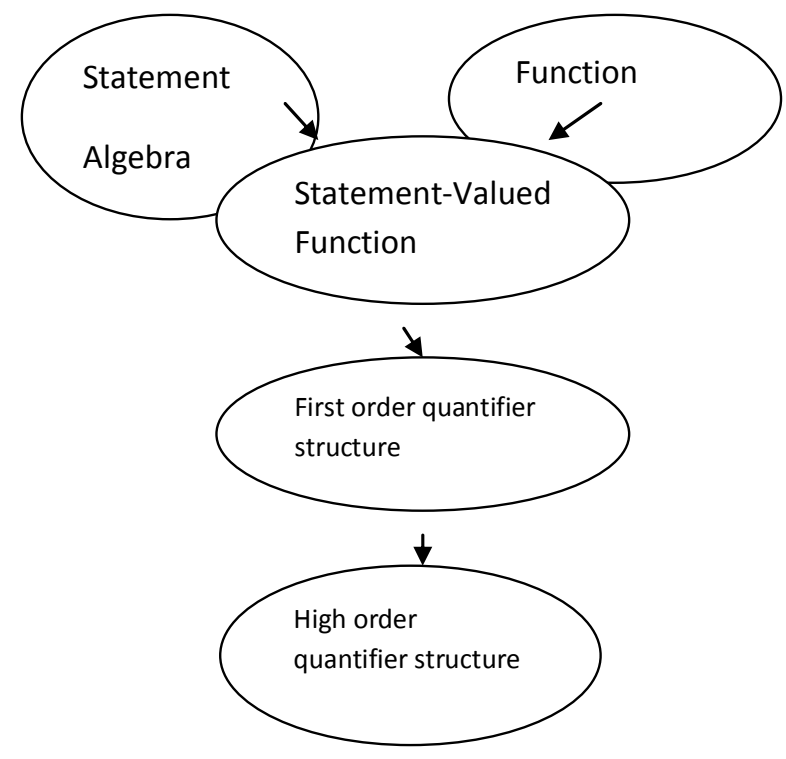

Figure 1. Genetic decomposition of quantifiers.

(either universal or existential), and are only applied to statement-valued functions with single variables. Transition to first order quantifiers is performed through the coordination of two previously structured processes. In other words, the statements obtained for various variable values are either combined with the universal quantifier or divided by the existential quantifier, such that only a single statement is obtained in the solution. Thus, the student internalizes (or encapsulates) the reiteration process of a variable in the domain to obtain a statement set in which a quantifier is used.

In second order quantifiers (which represent the next step), two different quantifiers are generally applied consecutively in statement-valued functions with two variables. To be able to structure the second order quantifier concept, first order quantifiers need to be included into a statement. This results in the elimination of a variable from the process. If the original statementvalued function has two variables, the object is dependent on the value of the other variable, and the scheme belonging to first order quantifiers can be reapplied to this statement-valued function. Thus, while analyzing statements that include second order quantifiers and two variables, it is possible to determine a student's ability to analyze the two quantifiers contained by the statements. In statement-valued functions with two variables, there is an inner quantifier on one of the variables, and an outer quantifier on the other variable. This new process, formed by the coordination of inner and outer quantifiers, should be included in order to obtain a single statement that will be a second order quantifier. When a third order quantifier expression is provided, the student can group the expression with two inner quantifiers, and once again apply a second order scheme to the outermost variable to obtain a defined statement. As before, this statement-valued function is included in order to obtain a single statement.

In conclusion, to obtain a quantifier of any other, this method is repeated as necessary and indeterminately.

\section{METHODS}

This study aimed to determine the mental structures developed by students in the process of forming quantifier concepts, and was designed as a qualitative study to be conducted within the context of the Abstract Mathematics course of the Elementary School Mathematics Teaching program.

\section{Participants}

In qualitative studies, the researcher directly spends time in the study area/field, meets with people within the context of the study, and utilizes the perspectives and experiences he/she acquired during the study in the analysis of the collected data (Yıldırım and Şimşek, 2003, p.23). The 97 students who attended the Abstract Mathematics courses during the 2012-2013 academic year constituted the participants of the study. The researcher of this study is the instructor of the said Abstract Mathematics course. According to Baki (2004), in the context of a mathematical study or problem-solving activity, mental structures are defined as images or words. It is thus possible to state that the students' mental structures regarding mathematical expressions that contain quantifiers are, in fact, visible on paper. Based on this, the problem form consisting of four open-ended questions that were developed by the researcher was first administered to the 97 students. Following the administration of these questions, the criterion sampling method (which is one of the purposive sampling methods) was used to select five students with whom interviews would be performed. Criterion sampling involves the review and evaluation of all cases that satisfy a set of pre-defined criteria. In this context, the criterion used in the selection of students to be interviewed was the students' ability to effectively understand the quantifier concept at a process level during the analyses performed by using the comprehension levels determined according to the APOS learning theory. The students' ability to understand the quantifier concept was determined based on data from the problem forms.

\section{Data collection tools and data collection process}

In this study, a problem form consisting of four open-ended questions and clinical interview techniques were used as data collection tools. The open-ended questions were prepared such that they assessed the students' understanding of the quantifier concept according to criteria selected from the literature.

In order to assess the students' ability to determine and express with symbols the truth values of open statements expressed in writing (verbally), by taking into account the truth values for statements obtained for each variable of the variable set; the first question of the problem form was prepared as follows:

Question 1: The open statements $p(x), q(x), r(x), s(x)$ and $t(x)$ (which are defined from a set of integers) are defined as follows:

$p(x): x>0$

$q(x): 2^{x}+1$ is a prime number.

$r(x): x$ is a perfect square.

$s(x): x$ is the sum of two prime numbers

$t(x): 4^{x} \geq x$ 
By writing them in a symbolic form, determine whether the expressions below are correct or incorrect.

a) If the $2^{x}+1$ value is a prime number when the $x$ value is an integer, then $x$ is a perfect square.

b) There is a natural number that is the sum of two prime numbers, and also a perfect square.

c) For the non-positive integer value, $4^{x}<x$ !

The second question of the problem form aimed to determine the reasoning used by students to interpret a first order quantifier structures that contained a universal quantifier. With this question, we investigated the extent to which the students were able to encapsulate first order quantifiers within the theoretical framework of APOS.

Question 2: $A \neq \emptyset$ Assume any $A \neq \emptyset$ set, and two statements $p$ and $q$ defined according to the $A$ set. Based on this information, evaluate the validity of the statement below:

$[(\forall x \in A, p(x)) \vee(\forall x \in A, q(x))] \Rightarrow[(\forall x \in A, p(x) \vee q(x))]$

Question 3 was asked to investigate the students' ability to determine the truth values of statement-valued functions provided in a symbolic form containing two or three quantifiers.

Question 3: $A=\{1,2,3,4\}$ Determine the truth values of the statements defined according to the $A=\{1,2,3,4\}$ set by writing down their verbal expressions, and explain your reasoning.
a) $\exists x, \forall y, x^{2}<y+1$
b) $\forall x, \exists y, x^{2}+y^{2}<12$
c) $\forall x, \forall y, \forall z x^{2}+y^{2}<2 z^{2}$
d) $\exists x, \exists y, \forall z x^{2}+y^{2}<2 z$

Question 4 was asked in order to assess the students' ability to determine the truth value of a written open statement that contains three quantifiers by writing the statement in a symbol form. Question 4 also assessed the students' ability to determine the truth value of an open statement with three quantifiers by coordinating it with three situations containing a single quantifier each.

Question 4: Assume that $p(x, y, z): x^{2}+y^{2}<2 x z$ is an open statement based on a set of integers. For each $x$ integer value, there is at least one $y$ integer value. As such, determine the truthvalues for each $z$ integer value by writing the $x^{2}+y^{2}<2 x z$ statement in a symbolic form, and explain your reasoning.

In qualitative studies, "triangulation" is one of the methods used to ensure validity and reliability (Yıldırım and Şimşek, 2003). To ensure the validity and reliability of this study, data diversification was performed with the problem form and the clinical interviews. In addition, to ensure the content validity of the tests prepared for this study, the researchers consulted an expert in the field. The reliability of the test was determined by using the dual-coding method developed by Miles and Huberman (1994). The data obtained by using the tests were analyzed qualitatively through coding by two experts in the field, one of whom was the author of this study. Based on this analysis, the reliability of the study was determined to be $83 \%$. This value demonstrated that the developed scale was suitable for consistently and reliability assessing the comprehension levels determined according to the APOS learning theory.

Following the qualitative analyses performed by coding the results of the tests that assessed the students' ability to form quantifier concepts, clinical interviews were used to collect further data. Clinical interview represents an interview technique that is frequently used in mathematics education, and which assists in determining the chain of thought of the students and in understanding their cognitive processes. Students who were included in the clinical interviews were selected among those whose cognitive levels were (at least) sufficient for forming first, second, and third order quantifier structures (as mentioned earlier in the "participants" section). Following the selection of students, clinical interviews were performed. The clinical interview questions were prepared with the assistance of experts' opinions. The final version of these questions is provided below. A pilot trial with the interview questions was performed with one of the students doing the Abstract Mathematics course.

Question 1: How would you define an "open statement"?

Question 2: Regarding the convergence set of triangles and rectangles, the following open statements are provided:

$p(x)$ : all sides of $x$ are equal.

$q(x)$ : all angles of $x$ are equal.

$r(x): x$ is an equilateral triangle.

According to the information above, describe whether the statement $\forall x[(p(x) \wedge q(x)) \Rightarrow r(x)]$ is correct or incorrect by expressing this symbolic statement in a verbal form.

Question 3: $p(x, y): x+y=9$ Assume that $p(x, y): x+y=9$ is an open statement based on a set of integers. According to this, evaluate the equivalence of the open statement $\forall x \exists y p(x, y)$ with the open statement $\exists y \forall x p(x, y)$.

Question 4: $\exists y \forall x p(x, y) \forall x \exists y p(x, y)$ What is the implication between the statements $\exists y \forall x p(x, y)$ and $\forall x \exists y p(x, y)$ ?

Question 5: Regarding the set of real numbers, determine the truthvalue of the following statement:

$[\forall x \forall y(x>0) \wedge(x>0)] \Rightarrow[\exists z(x z>y)]$

As described in the literature (Dubinsky, 1991), and as mentioned in the earlier sections of this paper, the main criteria for evaluating the structuring of first, second, and third order quantifier structures at a process level, and the assignments related to these criteria during the interview questions were as follows:

1. By generalizing the function scheme to the statement-valued function scheme, being able to determine the truth values of the statements obtained for each variable belonging to the set of statement-valued function variables (Questions 1 and 2).

2. Understanding second order quantifier structures (Questions 3 and 4).

3. Understanding third order quantifier structures (Question 5).

While seeking answers to these questions during the interviews, and depending on the responses of the students, simple questions such as "why?" and "how did you think of that?" were asked without guiding the students - in order to allow them to more comprehensively demonstrate their way of thinking.

Clinical interviews were performed not only to determine, based on the analysis results, if the cognitive level of students was sufficient to grasp only processes regarding quantifiers, and whether they were able to comprehend further aspects regarding quantifiers at an object or schemes level; but also to ensure data reliability through data triangulation. Prior to beginning the clinical interviews, the students were first informed regarding the purpose of the interviews, and the way in which they would be conducted. The students' consent for the interview procedures was also obtained prior to the interviews. During the interviews, the students were provided with a sheet to work with; while the students were verbally informed by the interviewer regarding the interview questions, they were also asked to use the worksheet to solve the 
questions. Furthermore, the students were asked not to erase any mistakes they made on the sheet, and to continue their work and calculations in the lines below. The interviews lasted an average of $40 \mathrm{~min}$, and were also recorded with an audio recording device. As the researcher was also the instructor of the students during the Abstract Mathematics course, the interviews were performed after the final exams of this course were completed in order to avoid any undesirable influence on the students during the interview process. The researcher also remained impartial throughout the study procedures.

\section{Analysis}

The study data were analyzed qualitatively by using the coding method, and the analyses were supported with information from the current literature. The analyses were performed according to the criteria regarding the determination of comprehension levels within the APOS framework. The test consisted of three categories, which were first, second, and third order quantifier structures. As such, the analysis of the results was also performed according to these three categories.

During the process of forming first order quantifier structures, the students were expected to form the structures listed below:

1. To combine and coordinate two or more statements by using logical connections.

2. To generalize the function scheme to the statement-valued function scheme.

3. To determine the truth value of statements obtained for each variable value belonging to the set of statement-valued function variables.

4. To determine quantifiers by coordinating statements with iterations regarding the statement-valued functions.

5. To encompass the process for first order quantifiers in order to obtain a single statement.

During the process of forming single order quantifier structures, students who could generalize the function scheme to the statement-valued function scheme, and determine the truth value of statements obtained for each variable value belonging to the set of statement-valued function variables were considered to have an action level comprehension of quantifiers. If the students were able to determine quantifiers by coordinating statements with iterations regarding the statement-valued functions, they were then considered to have a process level comprehension of quantifiers. If the students were able to obtain a single quantifier and single variable statement by determining the quantifiers, and by taking into account the truth values regarding the statement-valued function variables, the students were then considered to have achieved an object level comprehension. Such students could be considered to have internalized (or encapsulated) first order quantifiers.

Following the internalization of first order quantifiers, the students were expected to form the structures listed below when working with second order quantifiers:

1. To determine the internal and external quantifiers of statements that contain two different (and generally consecutive) quantifiers, and which are formed from two-variable statement-valued functions. 2. To determine the truth value of the statement by coordinating internal and external quantifiers.

3 . To encompass the process for second order quantifiers in order to obtain a single statement.

During the process of forming second order quantifier structures, students who could determine the internal and external quantifiers of statements that contain two different (and generally consecutive) quantifiers, and the truth value of the statement by taking into account the effect of the quantifiers on one another, were considered as having a process level comprehension of forming second order quantifiers. If the students could obtain two variable statement functions with two quantifiers through the coordination of internal and external quantifiers, the students were then considered to have an object level comprehension of second order quantifiers, and also to have internalized second order quantifier structures.

Finally, the students were expected to form third order quantifier structures by coordinating single order quantifier schemes for three quantifiers. Students who could group statements with three different quantifiers into two internal quantifiers, and employ the second order scheme to obtain a statement based on the most external variable were considered to have process level comprehension regarding the formation of third level quantifiers. If the obtained statement-valued function was (in a manner similar to previous structures) could be expressed as a three variable function three quantifiers, then the student was considered to have achieved object level comprehension of third order quantifiers, and to have internalized third orders quantifier structures.

\section{RESULTS}

The study results obtained in a question-answer format will be provided in this section in a descriptive manner. Based on the analysis results regarding the students' comprehension of the quantifier concept (which were support with information from the current literature), the developments observed among the students are detailed and explained below by using the data for the individuals students who were interviewed.

\section{Results regarding questions on first order quantifier structures}

The answers provided to the first question of the problem form, which were based on structures that formed in the students' minds within the APOS framework, are categorized and provided below.

Table 1 provides the results regarding the students' ability to write in symbol form an open statement expressed in writing by coordinating it with logical connections.

Table 1 shows that although $55 \%$ of the students were able to write open statements that were expressed verbally in symbol form, by coordinating them with logical connections, they were nevertheless unable to determine the quantifiers contained by the statement. The students were considered partially successful. On the other hand, $29 \%$ of the students were able to use logical connections to write statements that were expressed verbally in symbol form, and to identify the relevant quantifiers in the process. Based on these results, it is possible to state that the students had great difficulties in identifying quantifiers in statements that were expressed verbally.

The students' ability to determine the truth values of statements obtained for each variable belonging to the set of open statement variables, and their ability to identify the truth values of the open statements based on these reiterations are described in Table 2. 
Table 1. Ability to write in a symbol form by using logical connection.

\begin{tabular}{lcc}
\hline Ability to write in a symbol form by using logical connections & No of students & $\%$ \\
\hline Fully able & 28 & 29 \\
Partially able & 53 & 55 \\
Unable & 16 & 16 \\
\hline
\end{tabular}

Table 2. The ability to determine the truth value of each open statement.

\begin{tabular}{lcc}
\hline The ability to determine the truth value of each open statement & No of students & $\%$ \\
\hline Fully able & 28 & 29 \\
Partially able & 9 & 9 \\
Unable & 60 & 62 \\
\hline
\end{tabular}

Table 3. The distribution of the ability to determine universal quantifier and existential quantifier.

\begin{tabular}{lcc}
\hline Scope & Correct & $\%$ \\
\hline Universal Quantifier & 10 & 36 \\
Existential Quantifier & 18 & 64 \\
\hline
\end{tabular}

Among the students, $9 \%$ were able to determine the truth value of each statement obtained for every variable belonging to the set of open statement variables. However, they were not able to coordinate the statements obtained through reiterations, and were thus unable to identify the quantifiers. Therefore, it was considered that these students had only a partial understanding regarding the truth values of open statements. On the other hand, $62 \%$ of the students described the truth values of the open statements erroneously. According to the data in Table 2, $9 \%$ of the students were able to structure first order quantifier structures within the APOS theoretical framework at an action level. Furthermore, $29 \%$ of the students were able to determine the quantifier that needed to be part of the open statement expression by determining the truth values of the statements obtained for the variable values belonging to the set of open statement variables, and by identifying the relationship between the obtained statements. Based on the logical processes they used, these students were able to correctly determine the truth value of the open statement. Thus, $29 \%$ of the students were able to internalize (or encapsulate) the ability to form first order quantifiers, and to structure these quantifiers according to the APOS theoretical framework at an object level.

Table 2 also shows that $71 \%$ of the students were unsuccessful and had great difficulties in identifying universal or existential quantifiers in open statements that were expressed verbally. In addition, the students' open statements with single order quantifier structures were evaluated according to the types of quantifier they contained. Based on the analyses, it was determined that the students were more successful in forming first order structures that contained existential quantifiers than first order structures that contained universal quantifiers. The results are provided in Table 3.

\section{Results regarding the second question}

The students' ability to determine the truth value of compound statements belonging to a first order quantifier structure, and which are expressed in symbol form and contain a universal quantifier, is provided in Table 4.

As can be seen in Table 4 , only $5 \%$ of the students were able to correctly determine the truth value of the statement by taking into account the role of the universal quantifier in a compound statement with a first order quantifier structure and a universal quantifier. Among the students, $11 \%$ attempted to demonstrate the validity of the statement by using universal quantifiers and algebra. However, these students made various errors of reasoning when using algebra. Table 4 also shows that the large majority of the students determined the truth value of the compound statement incorrectly. The reasoning or ways of thinking that led students to these incorrect results are listed below:

Reasoning 1: If the statement $p(x)$ is incorrect for every value $x$ that belongs to the variable set (A) of the compound statement, then the " $\forall x \in A, p(x)$ " statement is incorrect as well.

Reasoning 2: Determining only the truth values for the open statement obtained by combining " $\mathrm{V}$ " and " $\Rightarrow$, without taking the universal quantifier into account.

Reasoning 3: Attempting to simplify the expression by applying symbolic logical operations on the compound 
Table 4. The distribution of the ability to determine the truth value of compound statements belonging to a first order quantifier structure.

\begin{tabular}{lcccccc}
\hline Scope & Correct & $\%$ & Partially correct & $\%$ & Incorrect & $\%$ \\
\hline $\begin{array}{l}\text { The ability to determine the truth value of a } \\
\text { compound statement with a first order quantifier } \\
\text { structure and a universal quantifier }\end{array}$ & 5 & 5 & 11 & 11 & 81 & 84 \\
\hline
\end{tabular}

Table 5. The distribution of the reasoning used by students who incorrectly interpreted the compound statements with a first order structure and a universal quantifier.

\begin{tabular}{lcc}
\hline Items & Students & $\%$ \\
\hline Reasoning 1 & 17 & 18 \\
Reasoning 2 & 23 & 25 \\
Reasoning 3 & 12 & 13 \\
Reasoning 4 & 12 & 13 \\
Reasoning 5 & 18 & 20 \\
Reasoning 6 & 10 & 11 \\
\hline
\end{tabular}

statement.

Reasoning 4: The student attempts to determine the truth value of the compound statement $\left[p\left(x_{0}\right) \vee q\left(x_{0}\right)\right] \Rightarrow$ $\left[p\left(y_{0}\right) \vee q\left(y_{0}\right)\right]$ for a $y_{0}$ value that differs from $x_{0}$ and $x_{0}$ (which, respectively, belong to the statements $p$ and $q$ from the set of variables $(A)$ ).

Reasoning 5: The students erroneously assume that the truth value will be 1 for the a $x_{0}$ value that belongs to the variable set of an open statement that includes the universal quantifier $(\forall$.

Reasoning 6: Determining $p$ and $q$ such that the truth value of expression " $\forall x \in A, p(x) \vee q(x)$ is 0 .

Based on the above categories, the distribution of the reasoning used by the students who incorrectly interpreted the compound statement with a first order structure and a universal quantifier is provided below in Table 5.

Table 5 shows that $25 \%$ of the students attempted to determine the truth value of the compound statement by disregarding the role of the universal quantifier, and by focusing instead on the symbolic logical connections" $\vee$ " and " $\Rightarrow$ " that were part of the compound statement. On the other hand, $20 \%$ of the students demonstrated the fourth type of reasoning. While attempting to identify the truth value of the compound statement expressed with symbols, these students erroneously attempted determine the truth value of the statement $\left[\left(p\left(x_{0}\right)\right) \vee\left(q\left(x_{0}\right)\right)\right] \Rightarrow$ $\left[\left(p\left(y_{0}\right) \vee q\left(y_{0}\right)\right)\right]$ by utilizing the $[(\forall x \in A, p(x)) \vee$ $(\forall x \in A, q(x))]$ statement a $x_{0}$ value belonging to the $A$ variable set of the compound statement; and for the $(\forall x \in A, p(x) \vee q(x))$ statement, a $y_{0}$ value belonging to the $A$ variable set that is different from the $x_{0}$ value. Students demonstrated the fourth type of reasoning could be considered as having problems in applying the function scheme to the statement-valued functions. Among the students, $11 \%$ attempted to determine the statements $p$ and $q$ (which are variables of the compound statement) in a manner that rendered the compound statement of the question invalid. In this case, although the student is aware of the role of the universal quantifier, he/she is not aware that he/she is providing a special example that turns the compound statement (expressed in symbols) into a valid statement.

According to the results in Tables 4 and 5 , it is possible to state that the students experienced difficulties in interpreting compound statements with a first order quantifier structure that contained a universal quantifier, mainly because they were not able, within the APOS framework, to structure the first order quantifier at an object level.

\section{Results regarding questions on second and third order quantifier structures}

Depending on the mental structures expected from the students while they formed second and third order quantifier structures in symbol form within the APOS framework, the answers to the third question of the problem form were classified as follows.

The students' ability to verbally express two and three variable open statements that are provided in symbol form is shown in Table 6.

In Table 6 , it can be seen that $92 \%$ of the students were able to express open statements with second and third order quantifier structures that were expressed verbally.

The results regarding the students' ability to determine the truth value of open statements expressed in symbol form that had two variables and two quantifiers in differing order are provided in Table 7.

Table 7 showed that $41 \%$ of the students were able to determine the truth value of open statements with second order quantifiers that had quantifiers in the existentialuniversal order. Furthermore, it was observed that $8 \%$ of the students had the ability to determine the truth value of two variable open statements that had quantifiers in the 
Table 6. The distribution of the students' ability to verbally express two and three variable open statements.

\begin{tabular}{lcc}
\hline Scope & Correct \% & Incorrect $\%$ \\
\hline Ability to verbally express second and third order quantifier statements & 89 & 8 \\
that are provided in symbol form & 92 & 8 \\
\hline
\end{tabular}

Table 7. The distribution of the students' ability to determine the truth value of open statements expressed in symbol form that had two variable and two quantifier.

\begin{tabular}{ccc}
\hline Scope & Correct & Incorrect \\
\hline$\exists \forall$ & 40 & 24 \\
$\forall \exists$ & 8 & 25 \\
\hline
\end{tabular}

Table 8. The distribution of the students' ability to determine the truth value of open statements expressed in symbol form that had three variable and three quantifier.

\begin{tabular}{ccc}
\hline Scope & Correct & Incorrect \\
\hline$\forall \forall \forall$ & 23 & 30 \\
$\exists \exists \forall$ & 10 & 34 \\
\hline
\end{tabular}

universal-existential order. In light of this data, it was observed that students were more successful in forming second order quantifier structures with the $\exists \forall$ order than the $\forall \exists$ order.

Table 7 also showed that $51 \%$ of the students were unable to determine the truth value of open statements with second order quantifiers that were expressed in symbol form. An evaluation of the solutions provided by the students to these problems revealed that they generally ignored/overlooked the ordering of the quantifiers in front of the variables. It was thus determined that the students attempted to find the truth value of the open statements expressed in symbol form by using their own opinions regarding the statement.

The results regarding the students' ability to determine the truth value of open statements expressed in symbol form that had three variables and three quantifiers in differing order are provided in Table 8.

Table 8 shows that $24 \%$ of the students were able to determine the truth value of an open statement with a third order quantifier structure that had an order of $\forall \forall \forall$, while $10 \%$ of the students were able to determine the truth value of an open statement with a third order quantifier structure that had an order of $\exists \exists \forall$. Thus, it can be seen that most students had difficulties in determining the truth value of structures with three quantifiers in different orders.

Table 8 also showed that $66 \%$ of the students were unable to determine the truth value of open statements with third order quantifiers that were expressed in symbol form. A review of the answers and solutions provided by these students to the problem form questions showed that they were unable to properly understand open statements with second order or third order quantifiers. When analyzing the quantifiers of open statements with three variables, most students overlooked the ordering of the quantifiers. As a result of this, they did not group quantifiers as external or internal. The students consequently failed to understand the open statements, and attempted to determine truth values of the symbolic expression based on their own opinions and perceptions.

When the results shown in Tables 7 and 8 are considered together, it can be seen that $49 \%$ of the students were able to form second order quantifier structures within the APOS theoretical framework, while $34 \%$ were able to form third order quantifier structures. Thus, the students had greater difficulty in forming third order quantifier structures than second order quantifier structures.

\section{Results regarding questions on verbally-expressed third order quantifier structures}

Depending on the mental structures expected from the students while they formed third order quantifier structures in symbol form within the APOS framework, the answers to the fourth question of the problem form were classified and evaluated as follows.

The students' ability to express verbally three variable open statements provided in symbol form is shown in Table 9.

Table 9 also showed that $73 \%$ of the students were unable to determine the truth value of statements with third order quantifiers that were verbally expressed.

Results regarding the students' ability to determine the truth values of verbally-expressed open statements with third order quantifier structures by analyzing one external and two internal quantifiers is shown in Table 10.

Table 10 shows that only $7 \%$ of the students were able to determine the truth value of verbally-expressed open statements with three variables by analyzing the quantifiers it contained. On the other hand, $93 \%$ of the students were not able to properly understand open statements with third order quantifier structures that were 
Table 9. The distribution of the students' ability to express verbally three variable open statements.

\begin{tabular}{|c|c|c|}
\hline Scope & Correct \% & Incorrect \% \\
\hline \multirow{2}{*}{$\begin{array}{l}\text { Ability to write in symbol form statements with third order quantifier structures that } \\
\text { are expressed verbally }\end{array}$} & 71 & 26 \\
\hline & 73 & 27 \\
\hline
\end{tabular}

Table 10. The distribution of the students' ability to determine the truth value of verbally-expressed open statements with third order quantifier structure.

\begin{tabular}{lcccc}
\hline Scope & Correct & $\%$ & Incorrect & $\%$ \\
\hline $\begin{array}{l}\text { The ability to analyze a statement with a third order quantifier structure by } \\
\text { using external and internal quantifiers }\end{array}$ & 7 & 7 & 90 & 93 \\
\hline
\end{tabular}

expressed verbally. These students attempted to determine the truth value of the written statement based on their own opinions regarding the statement. This $93 \%$ failure rate possibly resulted from the students' inability to form second and third order quantifier structures in their minds.

\section{Interview results}

Interviews were performed to ensure data diversification, and to thereby increase the reliability of the study. In addition to this, the interviews also served to determine the cognitive level of students. In other words, the interviews attempted to evaluate whether the interviewed students were at least at a process level, or whether they were at an object or scheme level.

Here, the interview students will be numbered from one to five. The analysis results for some of the students will be provided in detail, while similar results in other students will be described briefly.

\section{Results for the first student}

The student's answers to the problem form and the results provided by this student during the interview were in agreement with one another. Through the use of logical connections, the student was able to write open statements in symbol form with first order quantifier structures that were expressed verbally. During the interview, it was observed that the student was able to determine the truth value of the statement obtained for each variable value from the variable set. This indicated that the students were able to utilize reiterations obtained from the variable set, and effectively form mental processes to solve this problem. However, even if the student was able to determine the existential quantifier in open statements with first order quantifier structures that were expressed verbally, the student was nevertheless unable to determine the universal quantifier. Based on these results, it was concluded that the student had a process level understanding of first order quantifiers. The results from the interview with this student supported this conclusion regarding the student.

Interviewer: Could you give an example of an open statement?

Student: Yes... Hmmm... For example, the statement $x+2>5$ is an open statement. Here, we obtain the $4>5$ statement for $x=2$, which is an incorrect statement; and the $10>5$ statement for $x=8$. The truth value of this statement is one; in other words, it is correct...

Interviewer: What is the set of the variables for the open statement $x+2>5$ ?

Student: Ummm... the integer set...

Interviewer: Good... So, considering this example, how would you define this open statement?

Student: It is a statement that is correct for certain values, and incorrect for other values.

Interviewer: Could you please write the expression for the statement provided in Question 2?

Student: "For each $x$; if the sides and angles of $x$ are equal, then $x$ is an equilateral triangle."

Interviewer: So, what is the truth value of this statement? Could you explain your reasoning?

Student: The truth value of this statement is 1 . The reason for this is as follows: Assume that $x=A B C$ is an equilateral triangle. In this case, the statement of the question should be " $(p(A B C) \wedge q(A B C)) \Rightarrow r(A B C)$ " ; in other words, it should be "If all sides of $A B C$ are equal, and if all angles of $A B C$ are equal, then $A B C$ is an equilateral triangle." Given these conditions, the truth value of the statement is 1 , because of $1 \Rightarrow 1$. Now, let $x=D E F$ be an equilateral triangle. In this case, the statement of the question should be " $(p(D E F) \wedge$ $q(D E F)) \Rightarrow r(D E F)$ "; in other words, it should be "If all sides of DEF are equal, and if all angles of DEF are equal, then $D E F$ is an equilateral triangle." Given these conditions, the truth value of the statement is 1 , because of $0 \Rightarrow 0$. Since these two situations are valid for all triangles, and because the symbolic expression in the 
question is a universal quantifier, the truth value of the statement is 1.

Interviewer: So, could $x=A B C D$ be a square?

Student: I guess not. This is because triangles in which the sides and angles are all equal can only be equilateral triangles. And the statement mentions triangle specifically.

This interview showed that the student was aware of the role of universal quantifiers when attempting to determine the truth values of a symbolically expressed statement with a first order quantifier structure that included a universal quantifier. However, the student overlooked the variable set of the open statement, and took only the verbal expression of the statement into account. The student failed to note that if $x=A B C D$ were to be a square, then the truth value would have been 0 . This observation indicated that the student had difficulties in generalizing the function at hand to the scheme of the statement-valued function when forming a first order quantifier structure.

In the third and fourth questions that were about second and third order quantifier structures, the first student attempted to determine the truth value of the statements by using a set of symbolic logic rules, instead of grouping the quantifiers as external and internal. Based on this, it is possible to state that the fact that the student did not structure the first order quantifier according to the APOS theoretical framework prevented him from understanding second and third order quantifiers.

\section{Results for the second student}

According to the answers provided by the student to the questions regarding first order quantifiers in the problem form, it was determined that the second student had an object level understanding of first order quantifiers within the APOS theoretical framework. This observation was further supported by the answers the student provided to questions 1 and 2 during the interview.

The second student also demonstrated a rather good performance in structuring second and third order quantifiers within the APOS theoretical framework when answering the third question of the problem form. On the other hand, the interview showed that the student had many difficulties regarding second order quantifier structures. The second student had difficulties in interpreting new expression obtained by changing the sequence of quantifiers in an open statement with two variables and a second order quantifier structure. The interview during which the interviewer questioned the student on this subject is summarized below:

Interviewer: How do you interpret the expressions $\forall x \exists y p(x, y)$ and $\exists y \forall x p(x, y)$ ?

Student: The expression $\forall x \exists y p(x, y)$ means "For each xinteger value, there is at least one y integer value that allows the equation $x+y=9$ to be valid."

On the other hand, $\exists y \forall x p(x, y)$ means "There is at least one $y$ integer value for which every $x$ integer value allows the equation $y+x=9$ is valid."

Interviewer: Why did you write $+x=9$ ?

Student: Because in the expression $\exists y \forall x p(x, y)$, the sequence was: $\exists y \forall x$. For this reason, the equation must be $y+x=9$.

Interviewer: Do you think it should be $\exists y \forall x p(y, x)$ ?

Student: Yes... And because it is $\forall x \exists y p(x, y)$, the sequence of $\exists y \forall x$ would make $p(x, y)$.valid.

Interviewer: Are $p(y, x)$ and $p(x, y)$ different from each other?

Student: In ordered pairs, changing the sequence should also change the meaning of the mathematical expression. I know this, but since the integers are $x+y=y+x$; then $p(y, x)$ and $p(x, y)$ must be the same. Interviewer: So, what do you think about the truth value of the statement : $\exists y \forall x p(x, y) \Longrightarrow \forall x \exists y p(x, y)$ ?

Student: $H$ mmm... Since $\exists y \forall x p(x, y)$ and " $\forall x \exists y p(x, y)$ are the same statements, either $1 \Rightarrow 1$ or $0 \Rightarrow 0$ should be applicable depending on whether the statement is correct or incorrect. In both cases, the truth value of the statement : $\exists y \forall x p(x, y) \Longrightarrow \forall x \exists y p(x, y)$ would be 1 .

The second student was aware that changing the sequence of the quantifiers, or the quantifiers in front of the variables, would change the meaning of an open statement with second order quantifier structure. However, the student erroneously assumed that changing the sequence of the quantifiers would also change the sequence of the ordered pairs within the open statement. In addition, the student attempted to analyze the open statement without determining whether its quantifiers were internal or external quantifiers, by considering only the symbolic expression of the statement. Therefore, according to the APOS theoretical framework, the cognitive level of the student in forming second order quantifier structures could be considered as being at a process stage.

While forming third order quantifier structures, the student experienced problems similar to those he had when forming second order quantifier structures.

Interviewer: What would you say the truth value of the fourth question is?

Student: Hmmm... This compound statement has three quantifiers. As $\exists x \in R$ is a real number expression, let us say that $z=3$. Since the open statement is ordered as $\forall x \forall y$, the statement corresponding to $z=3$ would be correct for each value of $x$ and $y$. Then it should be $x=10$ and $y=45$. It that case, we obtain the statement $30>45$. This shows that the statement is incorrect.

During the interview, the student overlooked the sequence of the quantifiers in the statement with the third order quantifier structure $\forall \forall \exists$. As a result, he interpreted 
the role of the existential quantifier incorrectly. Based on these results, it can be said that the student had a process level understanding of third order quantifiers.

\section{Results for the third student}

The third student's level of cognitive development in forming first order quantifier structures was similar to that of the second student. However, the third student performed differently when forming second order quantifier structures. During the interview, it was noted that the student paid attention to the sequence of the quantifiers when analyzing two variable open statements with second order quantifier structure. The student also answered the question by determining the internal and external quantifiers.

Interviewer: Can you please verbally describe the expressions $\forall x \exists y x+y=9$ and $\exists y \forall x, x+y=9$ ? Student: The expression $\forall x \exists y, x+y=9$ means that "For every $x$ integer value, there is at least on integer $y$ that satisfies the equation $x+y=9$." The expression $\exists y \forall x, x+y=9$, on the other hand, means that "There is at least one $y$ integer value that satisfies the equation $x+y=9$ for every $x$ integer value."

Interviewer: So, what would you say about the equivalence between $\forall x \exists y x+y=9$ and $\exists y \forall x, x+y=$ 9

Student: Those two expressions are different from one another...

Interviewer: Could you explain why?

Student: Hmmm... For $x=3$, the only value that satisfies the equation $x+y=9$ : is $y=6$. For every integer value $x=x 0$ such as $x=4, y=5, x=7, y=2, x=13, y=$ -4 , etc... there is a corresponding integer value of $y o=9-x 0 \ldots \ldots$ In that case, the open statement $\forall x \exists y x+y=9$ is correct. On the other hand, considering the expression $\exists y \forall x, x+y=9$, there is a value $y_{0}$ for which the equation $x+y_{0}=9$ is satisfied by the integer value $\forall x$. This means that $x=7$ for $7+y 0=$ 9 , and $x=3$ for $3+y 0=9 \ldots \ldots \ldots$ and so on. Taking these two equations into account, $y 0=2=6$ turns out to be self-contradictory. This indicates that the statement is incorrect, and that its truth value is 0 . For this reason, $\forall x \exists y x+y=9$ and $\exists y \forall x, x+y=9$ are not equivalent... Interviewer: What kind of an implication could there be between $\exists y \forall x p((x, y)$ and $\forall x \exists y p(x, y)$ ?

Student: Hmmm... since the statement $\exists y \forall x p((x, y)$ is incorrect, and the statement $\forall x \exists y p(x, y)$ is correct, the statement $\exists y \forall x p(x, y) \Rightarrow \forall x \exists y p(x, y)$ is not possible. But the statement $\forall x \exists y p(x, y) \Rightarrow \exists y \forall x p(x, y)$ is possible. Interviewer: How did you determine whether the statements $\exists y \forall x p((x, y)$ and $\forall x \exists y p(x, y)$ were correct or incorrect?

Student: I just explained a few moments ago...
The third student was able to determine the external and internal quantifiers when analyzing statements with second order quantifier structures. By coordinating the external and internal quantifiers, and by determining the truth value of the open statement, the student was able to determine that they were not equivalent. This performance demonstrated that the student could structure second order quantifiers at an object level according to the APOS theoretical framework. However, when evaluating the implication between the open statements $\forall x \exists y p(x, y)$ and $\exists y \forall x p(x, y)$, the student was unable to consider $p(x, y)$ as a variable. This indicated that the student could not coordinate the function scheme and the algebra scheme.

Although the third student had internalized second order quantifier structures, he had difficulties when analyzing third order quantifier structures.

Interviewer: What is the truth value of the open statement?

$[\forall x \forall y(x>0) \wedge(y>0)] \Rightarrow[\exists z(x z>y)]$ which

defined according to a set of real numbers?

Student: Well... since $\exists z$, let us use $z=3 \ldots$ In the meantime, since we have $\forall x \forall y$, let us try to find if there are examples that make this statement incorrect.... Also, let us assume that $x=10$ and $y=45$. It that case, we obtain the statement $30>45$. This statement is incorrect. In that case, the statement is incorrect, and its truth value is 0 .

The student's analysis of the open statement with a third order quantifier structure did not include the analysis of the sequence of quantifiers in the statement. Thus, the student's analysis was performed by focusing only on the type of quantifiers, and the judgment of the statement. Based on this, it was observed that the student could not properly analyze and resolve the quantifiers within the open statement with third order quantifier structures. This demonstrated that the student could not structure third order quantifiers according to the APOS theoretical framework.

\section{Results for the fourth student}

The cognitive skills of the fourth student regarding the formation of first, second, and third order quantifier structures were generally similar to those of the third student. The main difference between the cognitive developments of the two students was that the fourth student was able to consider open statements with unknown values and second order quantifiers (such as $p, q, r \ldots)$ as functions. Thus, the student was able to reason by coordinating the function scheme with the algebra scheme. During the interview, it was observed that the student was able to verbally express a statement in written form. 
Interviewer: What is the implication between the statements $\forall x \exists y p(x, y)$ and $\exists y \forall x p(x, y)$ ?

Student: The set $A$ is the set for mathematics department students, while the set $B$ is the set for physics department students. Thus, let us define statement $p(x, y)$ with set $A \times B$ as " $\mathrm{x}$ likes $\mathrm{y}$." The expression $\exists y \forall x p(x, y)$ means "There is at least one physics department student who is liked by all of the mathematics department students." In that case, there is at least one physics department student who is liked by all mathematics department students. For this reason, the equation must be $\exists y \forall x p(x, y) \Rightarrow \forall x \exists y p(x, y)$. On the other hand, the expression $\forall x \exists y p(x, y)$ means "there is at least one physics department student who is liked by all mathematics department students." From this expression, we cannot say that there is at least one student who is liked by all mathematics department students. Then $\forall x \exists y p(x, y) \Rightarrow \exists y \forall x p(x, y)$ would not be possible.

The fourth student thus demonstrated that he was able to structure second order quantifiers at an object level according to the APOS theoretical framework. On the other hand, while analyzing a statement with three variables and three quantifiers, the student was unable to group the first two quantifiers to obtain a statement from the outermost variable. The dialogue between the interviewer and the student regarding the fifth question was as follows:

Interviewer: How would you determine the truth value of the following statement, which is defined from a real number set?

$[\forall x \forall y(x>0) \wedge(y>0)] \Longrightarrow[\exists z(x z>y)]$

Student: For every real number $x$ and $y$, there is at least on real number $z$ that satisfies $z>y$. In that case, and since $x>0$, we would obtain $x z>y$. In this case, the statement is correct.

Interviewer: What if we had $x \leq 0$ instead? Would that be possible?

Student: Since every $(\forall)$ in the statement is $x$, it would certainly be possible...

Interviewer: Then what would happen if we had $x \leq 0$ ?

Student: It that case... since $x \leq 0$, we would have $x z \leq y$. Thus, the compound statement would have $0 \Rightarrow 0$; in other words, it would have a truth value of 1 . The statement would thus be correct.

As was the case with the problem form, the student was not able to group the first two quantifiers of the statement and to coordinate the quantifier structures while analyzing the open statement with a third order quantifier structure. The analysis of the data from the interviews indicated that the student was able to structure first and second order quantifiers at an object level, and third order quantifiers at a process level.

\section{Results for the fifth student}

The fifth student demonstrated the best performance with both the problem form and in the interviews. It was observed that this student had an object level cognitive development regarding the formation of first order quantifier structures. While analyzing an open statement with second order quantifiers, the student could easily determine the external and internal quantifiers. As a result of this, the student was able to identify the truth values of the statements by coordinating the external and internal quantifiers. Thus, the fifth student was able to internalize first order quantifier structures. Furthermore, the student demonstrated an object level cognitive development regarding the formation of second order quantifier structures. An analysis of the students' approach towards open statements with third order quantifier structures indicated that the student could coordinate external and internal quantifiers, and that he could structure second order quantifier structures at an object level. The results of the interview further supported these observations.

Interviewer: How did you analyze the open statement in the fourth question, which has a third order quantifier? Can you explain?

Student: First of all, in the open statement

$[\forall x \forall y(x>0) \wedge(y>0)] \Rightarrow[\exists z(x z>y)]$ let us define the external quantifier as a universal quantifier, and the internal quantifiers as universal and existential quantifiers. Thus we can write this statement such as $\forall x \forall y \exists z,[(x>0) \wedge(y>0)] \Rightarrow(x z>y)$. Due to the principle regarding the sequence of real number sets, for each $y$ real number value, there is at least one $z$, such that $z>y$. In that case, the truth value of the statement $\forall y \exists z, z>y$ is 1 . If we consider the outermost universal quantifier, for any $x$ positive real number value, it will be $x z>y$, and the truth value of the statement will be 1 due to $1 \Rightarrow 1$. On the other hand, and $x$ negative real number value, we will have $x z<y$, and the truth value of the statement will be 1 due to $0 \Rightarrow 0$. In this case the statement is correct.

Interviewer: What would happen if we have $=0$ ?

Student: If we had $x=0$, the truth value of the statement $\forall x \forall y(x>0) \wedge(y>0)$ would be zero 0 . In such a case, the truth value of the compound statement would be 1 , since $0 \Rightarrow 0$ or $0 \Rightarrow 1$ would be applicable. The statement would thus be correct.

While working on the problem form and answering the interview questions, the student demonstrated the ability to analyze statements with third order quantifiers by group the quantifiers as external and internal. Thus, the student could correctly determine the truth value of the statements based on the analyses that he performed. The obtained results indicated that the student had an object level ability to form first, second, and third order 
quantifier structures.

\section{CONCLUSION AND RECOMMENDATIONS}

Quantifiers are the fundamental cornerstones of mathematical structures. The ability to give meaning to abstract and conceptual aspects of mathematics is directly related to the ability to comprehend expressions that contain quantifiers. In this context, it is important to teach quantifiers and their fundamental roles in mathematics during undergraduate mathematics education.

The current literature emphasizes the importance of forming mental structures with first and high order quantifiers, and of making transitions both from symbolic expression to verbal expressions and from verbal expression to symbolic expression. According to the data in Tables 6 and 9, it was observed that the students were more successful in performing transitions from symbolic form to verbal form than performing transitions from verbal form to symbolic form. In addition, a comparison of Tables 1 and 9 also revealed that when performing transitions from verbal/written form to symbolic form, the students were more successful with third order quantifier structures than they were with single order quantifier structures. The reason for this was possibly the fact that the quantifiers in open statements with third order quantifier structures were expressed more clearly in verbal/written form than the quantifiers in statements with first order quantifier structures. Furthermore, it was observed that students had difficulties in identifying the quantifiers within statements in case words such as "every, all, at least" (used to describe the universal and existential quantifiers) were absent from the verbal expression, especially in the case of first order quantifier structures. As a result of this, the students were not able to fully and correctly express the symbolic form of the relevant verbal expressions. Furthermore, it was observed that the students were able to determine the truth values of statements obtained by using each variable value belonging to the variable set of the open statement. Based on this, it was concluded that the students were able to effectively use statements and function schemes (which are the basic tools of logic) during the process of forming first order quantifier structures.

During the study, the students were more successful in determining the truth value of open statements with existential quantifiers within a first order quantifier structure than they were in determining the truth value of open statements with universal quantifiers. This was possibly due to the difficulties they had in understanding universal quantifiers in open statements expressed verbally with words such as "If... in case..." This finding is in parallel with the results of Epp's (2010) study. Based on this result, attempts were made to determine the reasoning used by students in interpreting mathematical expressions with universal quantifiers and first order quantifier structures, such as $(p \Rightarrow q) \wedge r$. In such expressions, the students generally overlooked the role of the universal quantifier, and formed their own opinions regarding the symbolic expression of the statement. Furthermore, the students had difficulties in realizing that $p, q$ and $r$ in the symbolic expression of the statement were actually variables. This was possibly due to the students' inability to analyze open statements by combining the statement algebra and the function schemes. It was noted that the students had a tendency to simplify the expression of the statement by using various rules. Based on this observation, it is possible to state that the students were unable to structure first order quantifier structures at an object level according to the APOS theoretical framework.

In the context of undergraduate mathematics courses, second and third order quantifier structures are used more frequently than first order quantifier structures. For this reason, the current study also attempted to determine how students give meaning to second and third order quantifier structures.

During the study, it was observed that the student generally overlooked the sequence of quantifiers when analyzing open statements with high order quantifier structures. As result, the students did not group quantifiers as external and internal when working on open statements with second and third order quantifier structures. On the other hand, the students had a greater difficulty in giving meaning to symbolic expressions with a particular variable set (such as $A=\{1,2,3,4\}$ ) and a universal-existential quantifier sequence $(\forall \exists)$ than expressions with a existential-universal quantifier sequence $(\exists \forall)$. This observation contradicts with the results of Piatek-Jimenez (2010). Furthermore, with a variable set represented by $p(x, y)$, the students experienced more difficulties in giving meaning to twovariable open statements with the $(\forall \exists p(x, y))$ expression than to two-variable open statements with the $(\forall \exists p(x, y))$ expression. Based on these results, it is possible to state that in statements that are included into the symbolic form of an expression (such as $p, q, r \ldots .$. ), changing the sequence of quantifiers led confusion among the students. In third order quantifier structures, the students had greater difficulty in giving meaning to mathematical expressions with different quantifier sequences than to mathematical expressions with the same quantifier sequence. Based on this observation, it is possible to state that changing the sequence of quantifiers in high order quantifier structures led to considerable difficulties for the students when attempting to form mental objects with these structures. This finding is in agreement with the results of Piatek-Jimenez (2010).

It is believed that gaining knowledge of the reasoning used by students when forming first and high order quantifier structures, and also of the difficulties they experience in the process, will be beneficial in organizing undergraduate-level mathematics teaching activities in 
manner that is more meaningful to the students. Performing educational activities in mathematics with greater knowledge of the importance of quantifiers will allow mathematics education to be better understood by the students, and will also strengthen the basis for mathematics learning. This, in turn, will assist students in better understanding the nature of mathematics taught in physics, engineering, and education faculties, and provide them with a better opinion on how to utilize or teach mathematics in their future occupations. In this context, mathematics instructors should plan their courses by taking into account the errors made by their students when forming quantifier structures, and attempt to remedy such errors by emphasizing the relevant aspects of quantifiers.

\section{Conflict of Interests}

The author has not declared any conflict of interests.

\section{REFERENCES}

Baki A (2004). Kavramsal ve işlemsel bilgi bağlamında lise öğrencilerinin cebir bilgilerinin karakterizasyonu. Türk Eğitim Bilimleri Dergisi 2(1):27-50.

Descartes R (2013). Yöntem Üzerine Konuşma. Birinci baskı, Say yayınları, İstanbul.
Dubinsky E (1997). On learning quantification. J. Comput. Mathematics Sci. Teach. 8(2):44-51.

Dubinsky E, Yiparaki O (2000). On student understanding of AE and EA quantification. In: E. Dubinsky, A. H. Schoenfeld, \& J. Kaput (Eds.), CMBS issues in mathematics education. Providence, RI: American Mathematical Society pp.239-289.

Dubinsky E (1991). Reflective Abstraction In Advanced Mathematical Thinking, in (D. Tall, ed) Advaced Mathematical Thinking Dordrecht: Kluwer pp.95-126.

Dubinsky E, Elterman F, Gong C (1998). The student's construction of quantification. For the Learn. Math. 8(2):44-51.

Miles MB, Huberman AM (1994). An Expanded Source Books Qualitative DataAnalysis, Second Edition, SAGE Publications, London.

Piatek-Jimenes K (2010). Students' Interprations of Mathematical Statements Involving Quantification. Math. Educ. Res. J. 20(3):41-56.

Yıldırım A, imek H (2003). Qualitative Research Techniques in Social Sciences, Seçkin Publications, Ankara.

Tall D, Chin ET (2002). University students' embodiment of quantifier. In: A.D. Cockburn \& E. Nardi (Eds.), Proceeding of the 26 th Conference of the International Group fort he Psychology of Mathematics Education, Norwich, UK. pp.273-280. 